\title{
Laboratórios de fabricação digital: uma revisão sistemática
}

\author{
Fab Labs: a systematic review
}

Thays Ramos Silva

Mestranda

Aluna, UFRGS, Porto Alegre, Brasil

Thays.ramos@icloud.com

\section{Fabio Pinto da Silva}

Doutor em Engenharia de Minas, Metalúrgica e de Materiais

Docente doDepartamento de Design e Expressão Gráfica (DEG/FA/UFRGS)

UFRGS, Porto Alegre, Brasil

Fabio.silva@ufrgs.br

\section{Evelise Anicet Ruthschilling}

Doutora em Informática da Educação

Docente do Departamento de Departamento de Artes Visuais (DAV/UFRGS) UFRGS, Porto Alegre, Brasil anicet@ufrgs.br

\section{RESUMO}

O objetivo deste artigo é mapear quais temáticas vêm sendo abordadas em produções científicas acerca de laboratórios de fabricação, e quais tipos de metodologias são empregadas nestes estudos, a partir de uma revisão sistemática de artigos e dissertações que tenham esse tipo de espaço como tema central. A busca se deu em bases de dados como Web of Science, SciELO, Scopus e Google Acadêmico (utilizado pela escassez de trabalhos desenvolvidos no Brasil nas outras bases de dados), incluindo trabalhos publicados entre 2014 e 2018, em português, inglês e espanhol. Foram analisados 26 artigos que continham os termos "Fab Lab" ou "Textile Lab" no título, palavras-chave ou resumo. Os resultados mostram que todos os trabalhos empregam uma abordagem qualitativa para coleta e análise de dados; sete diferentes eixos temáticos foram encontrados e a maioria dos artigos traz estudos sobre as relações de aprendizado no contexto Fab Lab.

Palavras-chave:Fab Lab; laboratórios de fabricação; revisão sistemática da literatura; Textile Lab; metodologias.

\section{ABSTRACT}

The purpose of this article is to map which themes have been approached about manufacturing laboratories, and what types of methodologies are used in these studies. To do so, a systematic review of articles and dissertations were employed. The search was done in databases such as Web of Science, SciELO, Scopus and Google Scholar (used for the shortage of works developed in Brazil in other databases), including works published between 2014 and 2018 in portuguese, english and spanish. 26 articles that contained the terms "Fab Lab" or "Textile Lab" in the title, keyword or abstract were analyzed. The results show that all the studies use a qualitative approach for data collection and analysis; seven different thematic axes have been found and most of the articles present studies on learning relationships in the Fab Lab context.

Keywords: Fab Lab; Fabrication laboratory; systematic review; Textile Lab; research methodology. 


\section{INTRODUÇÃo}

A indústria tem passado por uma série de modificações necessárias, o que ocorre tanto pela evolução rápida das tecnologias, quanto pelo descontentamento de uma parcela dos consumidores, que estão cada vez mais atentos a questões ambientais e sociais. Muito por isso, é possível constatar o surgimento de um movimento que visa o empoderamento do usuário e, como consequência, o surgimento de novos atores de inovação. Neste contexto, espaços como Fab Labs, que permitem ao mesmo tempo acesso a aparelhos de fabricação digital, surgem como expoentes neste processo de democratização do fazer (ANDERSON, 2012; BRUNO, 2017).

Apesar de ser um tema que vem sendo explorado em artigos científicos, muito em função da notória expansão da rede Fab Lab, há ainda muito a ser investigado sobre este fenômeno, e distintos vieses para conduzir estas investigações, como pretende demonstrar o presente artigo. Desta forma, o problema de pesquisa que o norteia foi definido como: quais são os temas mais recorrentes e os métodos de coletas e análises de dados mais utilizadas, em pesquisas acerca de laboratórios de fabricação digital (Fab Labs e Textile Labs)?

Para a condução desta pesquisa, optou-se pelo desenvolvimento de uma revisão sistemática da literatura, em uma mescla das técnicas propostas por Dresch, Lacerda e Antunes Júnior (2015) e Galvão e Pereira (2014).Quanto à dimensão desta revisão, ela pode ser classificada como ampla, por buscar a variedade de temas, e menos profunda, pelo mesmo motivo. Como a ideia também é mapear quais são os temas utilizados na análise destes espaços, nenhum critério foi utilizado para a definição de uma perspectiva específica de estudo. 0 artigo visa também contribuir com pesquisas de outros acadêmicos das áreas de design, arquitetura e engenharias, demonstrando quais eixos temáticos, no que se refere a trabalhos sobre estes laboratórios, carecem de trabalhos e quais caminhos, metodologicamente falando, já foram trilhados por outros pesquisadores. Tratando-se de um assunto incipiente, e considerando a ascensão do movimento maker, como corroboram Anderson (2012) e Fletcher (2008), com um livro e um capítulo, respectivamente, destinados ao tema e, que esse movimento não está ligado estritamente à academia, é interessante para as áreas de saber supracitadas que trabalhos de cunho científico ajudem a caracterizar o movimento, e um artigo de revisão sistemática, buscando reunir pesquisas afins, tende a favorecer esse contato. Para entender esse panorama, o presente trabalho tem por objetivo a avaliação de trabalhos científicos sobre Fab Labs e Textile Labs, buscando mapear eixos temáticos explorados com maior frequência, e investigar que tipo de técnicas de coleta e análise de dados são mais utilizadas para descrever o contexto dos laboratórios. 


\section{LABORATÓRIOS DE FABRICAÇÃo: Fab Labs e Textile Labs}

Nos últimos anos, o consumidor-usuário que, desde a primeira Revolução Industrial, esteve cada vez mais alienado dos meios e procedimentos de produção, passou a buscar um papel mais ativo, muito em função do descontentamento com a indústria convencional, que segue um modelo bastante poluidor e sem cuidado com sua mão-de-obra. Neste cenário, surgiram novos conceitos como maker, prosumer e de customização em massa. Novas tecnologias de fabricação digital, como o corte a laser e a impressão 3D, auxiliaram nesse novo e emergente processo de descentralização da indústria. A democratização ao acesso destes equipamentos se deu muito graças aos Fab Labs, criados com a ideia de permitir que se produza "quase tudo", com incentivo a conexão entre criadores de diferentes locais e abertura à comunidade. Com essa ideia de inúmeras possibilidades de fabricação, novos equipamentos começaram a ser integrados nestes espaços, e conceitos, como o de Textile Lab, que traz a ideia de fabricação têxtil e de vestuário, e Wet Labs, que tratam de biofabricação, foram surgindo como variantes destes laboratórios(ANDERSON, 2012; BRUNO, 2017).

De forma geral, um Fab Lab é um espaço de fabricação que emprega o conceito de prototipagem rápida (que pode servir tanto para facilitar o processo de prototipagem, quanto para possibilitar uma maior experimentação, considerando que utiliza processos de menor custo), agrupa um conjunto de máquinas com comando numérico computadorizado (CNC) como impressora 3D, fresadora, máquina de corte a laser, etc., e está ligado a uma rede mundial, a FabFoundation. Duas das bandeiras mais fortes desse tipo de laboratório são a colaboração e/ou compartilhamento de ideias e projetos, e é justamente por isso que a organização em uma rede mundial, composta visando a troca de experiências e colaboradores, é vista como mais importante do que a quantidade de máquinas que compõem o local. 0 primeiro Fab Lab foi criado em 2001, nos EUA, dentro do laboratório interdisciplinar Center for Bits and Atoms (CBA), sediado no Massachusetts Institute of Technology (MIT), sob o comando de Neil Gershenfeld, professor e coordenador do MIT Media Lab. A premissa para esse tipo de espaço era de democratizar o acesso à fabricação digital, assim como instruir e preparar seus frequentadores (EYCHENNE e NEVES, 2013; FABFOUNDATION, 2018).

Como citado anteriormente, variações dessa ideia de laboratórios digitais de fabricação foram surgindo, e entre elas, é possível destacar os Textile Labs, pela semelhança entre a organização dos laboratórios (que compõem uma rede) e pelo seu sucesso. A formação dessa rede de laboratórios se deu na Europa, berço da maioria dos Textile Labs, devido principalmente a duas iniciativas: 0 Textile and Clothing Business Lab (TCBL) e o Fabricademy. O TCBL é um ecossistema de negócios que alia empresas, laboratórios de inovação (os Textile Labs), prestadores de serviços e consultores de negócios, visando a transformação do cenário atual das 
indústrias têxtil e de moda. Esse projeto de pesquisa e inovação foi fundado pela União Europeia em julho de 2015, faz parte do programa Horizon 2020, envolve 13 países, e tem orçamento aproximado de 8 milhões de euros. Atualmente, a iniciativa apoia 33 Textile Labs, em 13 países da Europa, com maior concentração na Itália. Paralelamente, o Fabricademy é, ao mesmo tempo, uma rede e um curso destinados a ensinar sobre as relações entre o fazer têxtil e de moda e ferramentas de fabricação digital. Essa rede, que tem intersecções com o TCBL, tem atualmente 14 sedes, distribuídas entre Europa, Ásia, América do Sul e do Norte. O curso oferecido pela rede se apresenta em dois formatos: um bootcamp, versão condensada do curso em 5 dias, e uma versão de 6 meses, na qual os 3 primeiros meses são destinados a leituras semanais e experimentação dos materiais, e os 3 últimos ao desenvolvimento do projeto final, orientado por algum professor conforme área de afinidade. Esse último é bastante semelhante ao FabAcademy, modelo proposto pela FabFoundation, responsável pelos Fab Labs (TCBL, 2018; FABRICADEMY, 2018).

Considerando que ambos tipos de laboratórios são bastante recentes, especialmente os Textile Labs, que têm, em média, 5 anos, não são abundantes os trabalhos científicos que abordam o tema. Por este motivo, entende-se que a revisão sistemática é uma boa alternativa para traçar um panorama dos estudos da área, o que será melhor explanado na seção a seguir.

\section{METODOLOGIA}

0 método utilizado neste artigo é o de revisão sistemática da literatura, desenvolvida a fim de mapear temas que estão sendo estudados sobre laboratórios de fabricação no mundo todo, assim como verificar os tipos de metodologia mais empregadas nessas publicações. Segundo Dresch, Lacerda e Antunes Júnior (2015), a revisão bibliográfica é um estudo secundário que avalia e mapeia, de forma crítica, através de um método explícito e planejado, estudos primários que perpassam o problema de pesquisa. Pode ser descrita também como a atividade de compilar dados científicos sobre um tema, e deve ser abrangente e não tendenciosa, evitando o viés. Como benefício da elaboração da revisão, os autores da "Design Science Research", apontam o contato com boa parte do que se está desenvolvendo sobre um tema, a possibilidade de rejeitar, confirmar ou comparar resultados de investigações diferentes, e de encontrar lacunas que ainda possam ser exploradas em certos assuntos (DRESCH, LACERDA E ANTUNES JÚNIOR 2015; GALVÃO E PEREIRA, 2014).

Existem diferentes passos possíveis na elaboração de uma revisão bibliográfica da literatura. Galvão e Pereira (2014) citam oito, sendo: (1) elaboração da pergunta de pesquisa; (2) busca na literatura; (3) seleção dos artigos; (4) extração dos dados; (5) avaliação da qualidade metodológica; (6) síntese dos dados (metanálise); (7) avaliação da qualidade das evidências; e (8) redação e publicação dos resultados. Dresch, Lacerda e Antunes Júnior 
(2015) apresentam quatro diferentes possibilidades propostas por autores distintos, mais enxutas do que os oito passos citados anteriormente. Em comum, elas apresentam, mesmo que de maneira menos explícita, uma fase de definição da questão/problema de pesquisa, e uma etapa de síntese dos resultados. Na presente revisão, foi utilizada uma adaptação do método integrado, definido no "Design Science Research", sendo (1) definição da questão e do framework conceitual; (2) objetivos e questões; (3) estratégia de busca; (4) busca, elegibilidade e codificação; (5) avaliação da qualidade; (6) síntese dos resultados; e (7) apresentação do estudo. A avaliação de qualidade com atribuição de notas para os estudos, que consta como um dos passos para revisão, foi deixada de lado, pois não seria correto comparar artigos com objetivos tão distintos - considerando que é um dos objetivos desta revisão o contato com o maior número de abordagens sobre Fab Labs possível. Além disso, a apresentação do estudo aparece no item destinado às considerações finais do artigo, devido à sua função semelhante.

Foi acrescida, também, uma seção que descreve os objetivos e questões a serem analisadas para conferir a qualidade dos textos encontrados, avaliando se perguntas consideradas fundamentais os integram. Dresch, Lacerda e Antunes Júnior (2015) apontam que o tipo de questão a ser respondida influi bastante na definição de critérios e fontes de busca. Eles apresentam dois tipos possíveis de revisão: agregativa e configurativa. A primeira resulta de uma questão mais fechada. Nela os resultados da avaliação de estudos primários agregam para a obtenção de resultados, e geralmente partem de uma hipótese. Questões mais abertas, que serão avaliadas de forma mais abrangente, conduzem a uma revisão configurativa. Segundo os autores, o seu objetivo é "o arranjo de diversos resultados individuais em uma renderização teórica coerente" (DRESCH, LACERDA E ANTUNES JÚNIOR, 2015, p. 147). Como a ideia desta revisão é buscar um panorama amplo, a revisão configurativa é mais adequada, porque consegue prover uma ideia mais geral do que vem sendo produzido, além do fato de o estudo não ter sido guiado por nenhuma hipótese.

\subsection{Definição Da Questão e Framework Conceitual}

Diferentes espaços de fabricação digital, em especial os Fab Labs, vem sendo estudados cada vez mais pela comunidade científica. As abordagens destes trabalhos são as mais diversas, desde trabalhos que exploram a característica pedagógica destes laboratórios a estudos que avaliam seu impacto socioeconômico. As questões principais da presente revisão literária são acerca das técnicas de coleta e análise de dados utilizadas nestes trabalhos, assim como um mapeamento detalhado de temas explorados. Com isso, a ideia é que o presente artigo auxilie pesquisadores interessados no tema a identificar lacunas a serem exploradas e quais estratégias de coleta e análise de dados mais adequadas.

Para explanar como esta questão será revisada, conforme o "Design Science Research", foi desenvolvido um framework conceitual, que 
demonstra, de forma esquemática, como será realizada a revisão (DRESCH, LACERDA E ANTUNES JÚNIOR, 2015). A direção utilizada para a realização da pesquisa assemelha-se com o esquema da figura 1.

Figura 1: Framework conceitual.

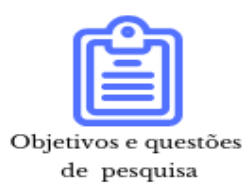

de pesquisa

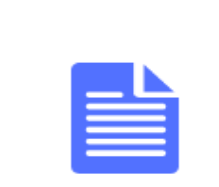

Análise de resumo 26 trabalhos

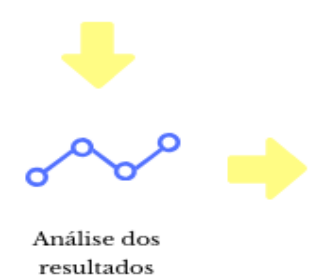

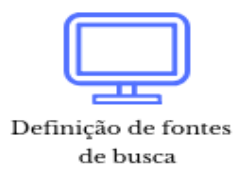

de busca

I N I C I ALIZ A ÇÃo

\section{A P LIC A Ç Ã O}

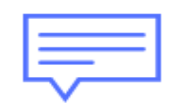

Análise de título

26 trabalhos

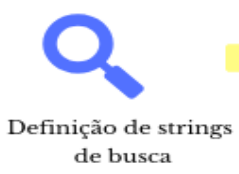

de busca

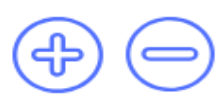

Definição de critérios de inclusão e exclusão

\section{ANÁLISE E SínTESE}

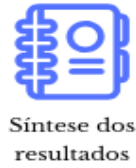

Fonte: elaborado pelos autores.

Os aspectos citados no framework, como fontes de busca, critérios de inclusão e exclusão, etc. serão apresentados nas seções a seguir.

\subsection{Objetivos e Questões analisadas}

A revisão pretende compreender o que já foi desenvolvido acerca de laboratórios de fabricação digital (que envolvam a produção de produtos de moda ou não), no Brasil e exterior, buscando além de um mapeamento de temas e fontes relevantes para o desenvolvimento deste estudo, verificar as formas de coleta e análise de dados utilizadas. As questões definidas para a pesquisa sobre os Fab Lab e Textile Labs foram:

C1 - Qual aspecto sobre o Fab Lab/Textile Lab é abordado?

C2 - Que tipo(s) de instrumento(s) metodológico(s) foi/foram utilizado(s) para

coleta de dados? 
C3 - Que tipo(s) de instrumento(s) metodológico(s) foi/foram utilizado(s) paraanálise de dados?

C4 - Qual foi a forma de apresentação dos resultados (texto, criação de diretrizes, workflow, etc.)?

\subsection{Fontes e Estratégias de Busca}

Em um primeiro momento, a busca se deu em bases de dados como a Web of Science, SciELO e Scopus. Devido à escassez de resultados acerca da intersecção entre fabricação de moda e Fab Labs, nas primeiras buscas, assim como de trabalhos em português que pudessem oferecer um panorama do tema no Brasil, optou-se por estender as buscas ao Google Acadêmico. Ainda sob esse critério, houve a flexibilização das buscas também para dissertações que versassem sobre essa relação.

Quanto as strings de busca, em relação aos laboratórios de fabricação foram utilizados: "Fab Lab" OR "Textile Lab" OR "FabLab" OR “TextileLab" OR "Fabrication Laboratory". Considerando que se trata de um tema novo, houve a opção por termos de busca mais genéricos, a fim de obter-se o maior número possível de resultados. Os termos foram pesquisados também com diferentes formas de grafia, pois foi constatado que houve uma quantidade diferente de resultados entre a busca por "Fab Lab" e "FabLab", por exemplo.

\subsection{Busca, Elegibilidade e Codificação}

Tendo em vista que as questões a serem investigadas estão relacionadas a um modelo de espaço de manufatura digital com pouco mais de 15 anos (podendo ser ainda mais recente, se tratando dos Textile Labs), o primeiro critério a ser definido envolve a data das publicações. Além disso, entendeu-se como mais relevante à avaliação de artigos em português, para que se construa uma ideia do que está sendo desenvolvido no Brasil, em inglês, por ser considerado um idioma universal, em que provavelmente a maiorias dos artigos enviados para periódicos de renome estarão redigidos, em espanhol, por ser a língua oficial de uma grande quantidade de países e possibilitar o contato com trabalhos realizados em países vizinhos.

Sendo assim, foram considerados como critérios de inclusão a) artigos publicados no período de 2014 a 2018, b) a disponibilidade dos trabalhos em língua portuguesa, inglesa ou espanhola. Como critérios de exclusão: a) trabalhos que se refiram apenas a uma das tecnologias presentes em um Fab Lab (apenas impressão 3D, apenas corte a laser, etc.), b) a ausência dos termos Fab Lab ou Textile Lab no título ou palavras-chave dos trabalhos.

\section{SÍNTESE DOS RESULTADOS}

Seguindo os passos apresentados anteriormente no framework conceitual, na busca por palavras-chaves foram encontrados 707 resultados, 
sendo 163 deles encontrados na plataforma Scopus, 271 na Web of Science, 11 na Scielo e 262 no Google Acadêmico. Após a fase de análise de título, foram selecionados 26 trabalhos, utilizando os critérios de inclusão e exclusão já citados, e esse número foi mantido após a análise dos resumos.

Foi possível perceber que, após uma fase de aplicação de filtros, muitos artigos foram classificados como inadequados, de alguma forma. Um dos motivos para a exclusão dos trabalhos foi a falta de relação dos achados com os objetivos da revisão - o string "Fabrication laboratories", por exemplo, apresentou em sua maioria resultados que divergiam bastante da ideia apresentada na seção 2 .

Após a análise dos artigos e dissertações, que envolvem, de alguma maneira, o estudo de laboratórios de fabricação digital, e com base nas questões apresentadas na seção 3.2, o quadro 1 foi elaborado, trazendo a síntese das respostas. Os trabalhos estão apresentados em ordem cronológica e alfabética, e estão identificados como artigo ou dissertação.

Quadro 1: trabalhos levantados na revisão, classificados por título/autores, tipo (dissertação/artigo), e ano de publicação.

\begin{tabular}{|c|c|c|}
\hline Título/autores & Tipo & Ano de publicação \\
\hline $\begin{array}{l}\text { Moda e fabricação digital em um contexto Fab Lab: Equipamentos, } \\
\text { métodos e processos para o desenvolvimento de produtos (BASTOS) }\end{array}$ & Dissertação & 2014 \\
\hline $\begin{array}{l}\text { The FAB LAB Network A Global Platform for Digital Invention, } \\
\text { Education and Entrepreneurship (STACEY) }\end{array}$ & Artigo & 2014 \\
\hline $\begin{array}{l}\text { Open Production: Chances for Social Sustainability in Manufacturing } \\
\text { (BASMER) }\end{array}$ & Artigo & 2015 \\
\hline $\begin{array}{l}\text { Possibilidades e Desafios de Um Espaço Maker Com Objetivos } \\
\text { Educacionais (BORGES, PERES, CATILHO, FAGUNDES) }\end{array}$ & Artigo & 2015 \\
\hline $\begin{array}{l}\text { How do fab-spaces enable entrepreneurship? Case studies of } \\
\text { 'makers' - Entrepreneurs (MORTARA, PARISOT) }\end{array}$ & Artigo & 2016 \\
\hline $\begin{array}{l}\text { Lite Maker: Um Fab Lab Móvel para Aplicação de Atividades Mão na } \\
\text { Massa com Estudantes do Ensino Básico (SANTANA et al.) }\end{array}$ & Artigo & 2016 \\
\hline $\begin{array}{l}\text { Maker Cultures and the Prospects for Technological Action } \\
\text { (NASCIMENTO, PÓLVORA) }\end{array}$ & Artigo & 2016 \\
\hline $\begin{array}{l}\text { O uso da prototipagem e fabricação digital no ambiente Fab Lab } \\
\text { (OLIVEIRA) }\end{array}$ & Dissertação & 2016 \\
\hline
\end{tabular}




\begin{tabular}{|c|c|c|}
\hline Bringing optics to Fab Labs in Europe (ADAM, URBACH) & Artigo & 2017 \\
\hline $\begin{array}{l}\text { Desenvolvimento e implantação de um Fab Lab: Um estudo teórico } \\
\text { (AGUIAR et al.) }\end{array}$ & Artigo & 2017 \\
\hline $\begin{array}{l}\text { Diversity in FabLabs: Culture, Role Models and the Gendering of } \\
\text { Making (VOIGT, UNTERFRAUNER, STELZER) }\end{array}$ & Artigo & 2017 \\
\hline Fab City com enfoque em economia circular (URRUTIA PINTO et al) & Artigo & 2017 \\
\hline $\begin{array}{l}\text { Fab Labs as Platforms for Digital Fabrication Services: A } \\
\text { LiteratureAnalysis (SAVASTANO et al.) }\end{array}$ & Artigo & 2017 \\
\hline $\begin{array}{l}\text { Fab Lab Lisboa: when a Municipality Fosters Grassroots, } \\
\text { Technological and Collaborative Innovation GAEIRAS) }\end{array}$ & Artigo & 2017 \\
\hline $\begin{array}{l}\text { Fab labs network in developing countries: Knowledge spillover } \\
\text { effects or managing technology development within the scarcity } \\
\text { economy? (VIEIRA, BRESCIANI, SANTOS) }\end{array}$ & Artigo & 2017 \\
\hline Make to learn: invention through emulation (BULL et al.) & Artigo & 2017 \\
\hline $\begin{array}{l}\text { Making "Making” Critical: How Sustainability is Constituted in Fab } \\
\text { Lab Ideology } \\
\text { (KOHTAL) }\end{array}$ & Artigo & 2017 \\
\hline $\begin{array}{l}\text { O Design dos Makerspaces e dos Fab Labs no Brasil: um } \\
\text { mapeamento preliminar(COSTA, PELEGRINI) }\end{array}$ & Artigo & 2017 \\
\hline $\begin{array}{l}\text { Perspectivas para a formação docente universitária com aspectos } \\
\text { Makers (FROSCH, ALVES) }\end{array}$ & Artigo & 2017 \\
\hline $\begin{array}{l}\text { Academic FabLabs for industry 4.0: Experience at University of } \\
\text { Naples Federico II(AGRISANI et al.) }\end{array}$ & Artigo & 2017 \\
\hline $\begin{array}{l}\text { A insustentável neutralidade da tecnologia: o dilema do Movimento } \\
\text { Maker e dos Fab Labs (CAMPOS, DIAS) }\end{array}$ & Artigo & 2018 \\
\hline $\begin{array}{l}\text { Design and Innovation Learning: Case Study in North African } \\
\text { Engineering Universities Using Creativity Workshops and } \\
\text { Fabrication Laboratories (BEM REJEB e ROUSSEL). }\end{array}$ & Artigo & 2018 \\
\hline $\begin{array}{l}\text { FabLab global survey: Characterization of FabLab phenomenon } \\
\text { (RUIZ, ACEBO) }\end{array}$ & Artigo & 2018 \\
\hline $\begin{array}{l}\text { Makers and clusters. Knowledge leaks in open innovation networks } \\
\text { (GIUISTI, ALBERTI, BELFANTI) }\end{array}$ & Artigo & 2018 \\
\hline O movimento maker: enfoque nos Fab Labs brasileiros (PINTO et al.) & Artigo & 2018 \\
\hline $\begin{array}{l}\text { Strategic knowledge management a digital environment: Tacit and } \\
\text { explicit knowledge in Fab Labs (MARAVILHAS, MARTINS) }\end{array}$ & Artigo & 2018 \\
\hline
\end{tabular}

Fonte: elaborado pelos autores. 
É possível notar que a maioria dos trabalhos analisados se utiliza de abordagem qualitativa para desenvolvimento dos estudos, tanto no momento de coleta de dados, quanto na análise dos mesmos. Todos os trabalhos apresentam uma fase preliminar de revisão bibliográfica e documental - por se tratarem de assuntos recentes, que não estão tão presentes em livros, além de dados que se alteram rapidamente, como por exemplo, o número de Fab Labs ao redor do mundo. Alguns artigos, como o de STACEY (2014), ADAM e URBACH (2017) e BULL et al. (2017) não indicam quais ferramentas de coleta de dados foram utilizadas para o desenvolvimento do estudo; os trabalhos de GAEIRAS (2017) e AGRISANI et al. (2017), não apresentam sequer uma seção destinada a apresentação da metodologia. Dois dos estudos se tratam de revisões sistemáticas da literatura: o trabalho de SAVASTANO et al. (2017) com pesquisas restritas a publicações em journals, que busca elucidar questões sobre o surgimento dos Fab Labs e seu status atual, e o trabalho de AGUIAR el al. (2017), que objetivava tipificar os laboratórios e definir requisitos para a criação de um laboratório de fabricação.

As ferramentas de coleta, citadas na literatura sobre estudo de caso, mais presentes nos trabalhos analisados nesta revisão, em ordem são: revisão bibliográfica ou documental, entrevistas, observação direta, questionários, observação participante, revisão sistemática da literatura, e grupo focal. Foram considerados aqui apenas os trabalhos que trouxeram exclusivamente os achados da fase de revisão bibliográfica para a conclusão. Como um dos objetivos da revisão era produzir um panorama de metodologias empregues para o estudo dos laboratórios de fabricação, o quadro 2 foi desenvolvido para apresentar de forma concisa esses resultados.

Quadro 2: classificação dos trabalhos estudados conforme sua natureza de coleta e análise de dados.

\begin{tabular}{|c|c|c|}
\hline Natureza do estudo & Ferramenta de coleta utilizada & № de trabalhos \\
\hline \multirow{2}{*}{ Quantitativa } & $\begin{array}{l}\text { Entrevista - método de análise: } \\
\text { Estimativas de Densidade Kernel }\end{array}$ & 1 \\
\hline & Questionário online & 2 \\
\hline \multirow{6}{*}{ Qualitativa } & Entrevista & 8 \\
\hline & Questionário & 4 \\
\hline & Observação direta & 7 \\
\hline & Observação participante & 3 \\
\hline & Pesquisa documental & 9 \\
\hline & Revisão sistemática da literatura & 2 \\
\hline
\end{tabular}


Fonte: elaborado pelos autores.

Três dos artigos levantados- VOIGT, UNTERFRAUNER, STELZER(2017); COSTA, PELEGRINI (2017);RUIZ, ACEBO (2018) - utilizam formas de análise quantitativas para complementar seus achados. Entre eles, dois (COSTA, PELEGRINI, 2017;RUIZ, ACEBO, 2018)se utilizam de surveys, buscando uma amostra maior para coleta de dados. Os três estudosse enquadram na definição de abordagem mista, que segundo Creswell (2007) é uma abordagem que combina ou associa as formas qualitativas e quantitativas.

Se foram três os artigos que não definiram as formas de coletas de dados (não incluindo os trabalhos que não incluíam uma seção de procedimentos metodológicos), o número de estudos que não deixou claro a forma de análise utilizada foi ainda maior, totalizando 14 artigos. Esse achado dificulta a resolução do artigo de demonstrar ferramentas interessantes para pesquisas futuras. As formas de análise de dados relacionadas foram: análise de conteúdo (BASTOS, 2014; OLIVEIRA, 2016; VIEIRA, BRESCIANI, SANTOS, 2017, MARAVILHAS, MARTINS (2018); uma combinação entre a ferramenta MAXQDA2 (qualitativa) e Densidade de Kernel (quantitativa) foi utilizada por Voigt, Unterfrauner, Stelzer (2017); a ferramenta de Interacionismo simbólico (SI) foi empregada no trabalho de Kohtal (2017); Giusti, Alberti e Belfanti (2018) utilizaram o método de Análise de redes sociais (SNA) e o software de análise UNICET 6 para análise de dados em rede. Os autores utilizaram ainda a tipologia de Giuliano e Bell (2015) e Fonti (2002) buscando verificar a multiciplicidade nos fluxos de conhecimento; este foi o artigo com maior detalhamento e número de técnicas de análise de dados dentre os levantados.

Para auxiliar no mapeamento de quais temas estão sendo explorados em trabalhos científicos acerca dos Fab Labs, o gráfico presente na figura 2 foi construído.Sete diferentes eixos temáticos foram encontrados. A maioria dos artigos estuda relações de aprendizado no contexto Fab Lab.Em seguida, aparecem os artigos que se propõem a conceituar, caracterizar e mapear a rede de laboratórios. Entre os 26 trabalhos levantados, apenas um se relaciona à produção de moda, e tem como objetivo relacionar as metodologias de Design de moda com o que é desenvolvido nestes espaços.

Se os Textile Labs encontraram apenas uma correspondência, os Wet Labs não foram citados em nenhum dos trabalhos levantados, apesar da biofabricação, bastante relacionada a questões ambientais, ter ganhado espaço em iniciativas como a Fabricademy e ter um espaço reservado em alguns laboratórios pelo mundo. 
Figura 2: temas explorados nos trabalhos que compõem a revisão.

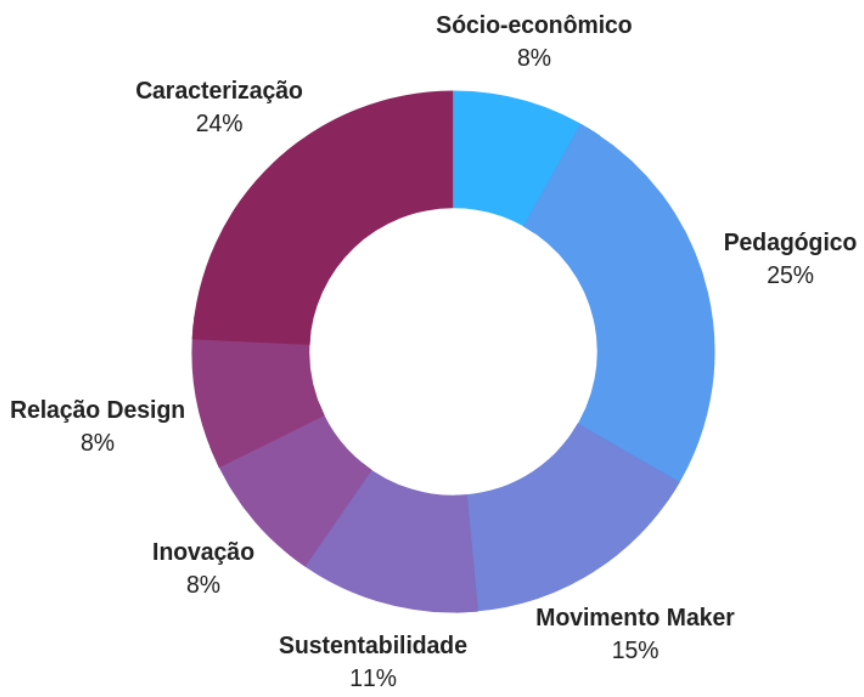

Fonte: elaborado pelos autores.

Relações socioeconômicas, a questão da inovação e a relação destes laboratórios com o design, totalizando cada $8 \%$ do total, despontam também como assuntos a serem explorados. A relação entre esses laboratórios e a sustentabilidade, apontada anteriormente como um dos vetores para a criação destes espaços, também foi pouco explorada.

Considerando que um dos fatores de inclusão dos artigos na revisão foi o ano de publicação, a figura 3 foi construída para demonstrar como os 26 artigos/dissertações estão distribuídos entre os anos de 2014 e 2018.

Figura 3: artigos conforme ano de publicação.

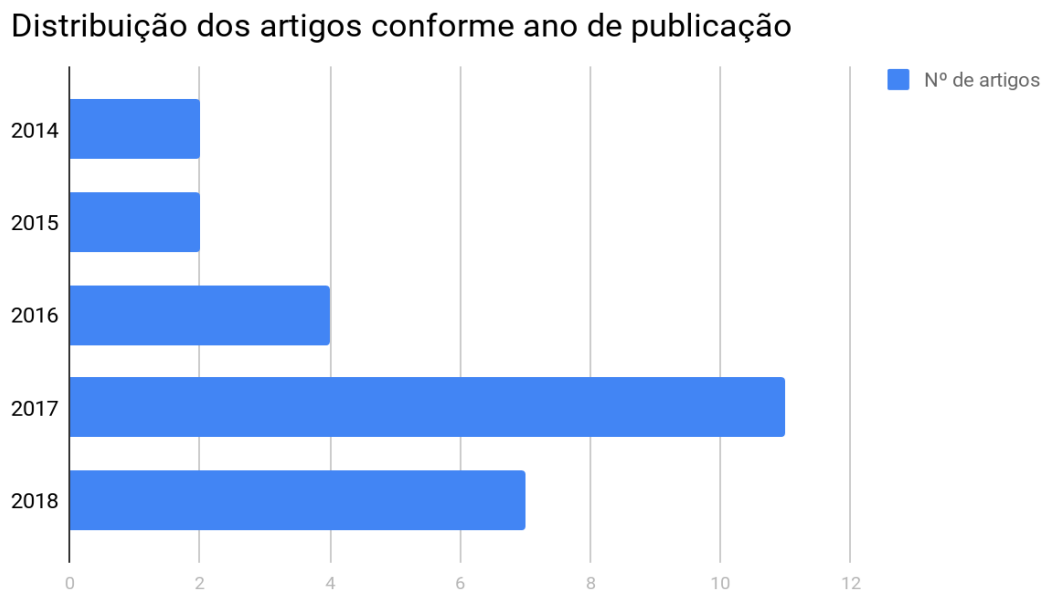

Fonte: elaborado pelos autores. 
Mais da metade dos artigos selecionados (tendo em vista os critérios de inclusão e exclusão citados anteriormente) foram publicados nos últimos dois anos (18 artigos).Esse dado pode demonstrar que a quantidade de trabalhos sobre o tema (artigos, dissertações e teses) vem crescendo, mesmo que o tipo principal de laboratório investigado, o Fab Lab, tenha sua origem há 17 anos.

É importante observar que a coleta de dados para o presente artigo foi finalizado em setembro de 2018, então o levantamento não contém a totalidade dos artigos publicados no ano. 0 único trabalho analisado que envolve Textile Labs foi desenvolvido em 2014, no início da rede de maior proeminência deste tipo de laboratório, a Fabricademy, e considerando o dinamismo destes espaços, é provável que mudanças tenham ocorrido nos últimos cinco anos e novas pesquisas se tornem necessárias. São cinco as pesquisas relacionadas à caracterização do movimento, uma de 2014, três de 2017 , e uma de 2018, o que pode demonstrar que mesmo que o surgimento dos espaços date de quase duas décadas, ainda perceba-se a necessidade da caracterização dos laboratórios, provavelmente pela questão citada anteriormente da plena expansão e constante mudanças que acontecem nos espaços. Os artigos acerca do movimento maker(6), datam dos últimos três anos, o que pode indicar um interesse crescente sobre o assunto.

\section{CONSIDERAÇÕES FINAIS}

Como apontado anteriormente, o objetivo do estudo era mapear quais eixos temáticos vem sendo abordados em publicações sobre Fab Labs e Textile Labs, e investigar, nos trabalhos analisados, formas de coleta e análise de dados mais comumente utilizadas. Foi possível constatar que são escassos os trabalhos sobre Textile Labs, e que há ainda bastante a ser explorado sobre o tema. Devido a esse fator de novidade, a pesquisa em outras fontes de levantamentos, além de livros, torna-se bastante comum.

Foi possível constatar,através da primeira etapa de busca em periódicos, que existem mais trabalhos acerca das tecnologias empregues nos laboratórios de fabricação digital (como impressão 3D, por exemplo), do que sobre esses espaços de produção. Isso pode indicar que a prática maker e seus espaços ainda estão um pouco descolados da comunidade científica.

A abordagem qualitativa, tanto para a fase de coleta, quanto de análise de dados, foi utilizada, mesmo que não exclusivamente, na totalidade dos trabalhos. Pôde-se observar que as coletas através dos diferentes tipos de observação (direta e participante) são bastante empregadas nestes estudos, muito em função da escassez de fontes bibliográficas. Isso acontece porque vários autores de metodologia de pesquisa, como Yin (2001), destacam a coleta através de observação para o estudo de fenômenos sem um relato histórico, ou seja, relativamente novos, como é o caso dos trabalhos levantados neste artigo. 0 uso maior da abordagem qualitativa pode ser explicado também pela profundidade buscada nos artigos, que optaram, em 
sua maioria, por se aprofundar nos relatos de um/alguns laboratório(s) específico(s), ao invés de buscar um panorama amplo da rede Fab Lab.

\section{REFERÊNCIAS BIBLIOGRÁFICAS}

ADAM, Aurèle. ZUIDWIJK, Thim. URBACH, Paul. Bringing optics to Fab Labs in Europe. 14th Conference on Education and Training in Optics and Photonics: ETOP 2017. CCC code: 0277-786X/17/\$18 - Disponível em: <doi: 10.1117/12.2281969>. Acesso em: 23 jun. 2018.

ANGRISANI, Leopoldo et al. Academic Fab Labs for industry 4.0: experience at University of Naples Federico II. IEEE Instrumentation \&Measurement Magazine. Disponível em: < https://ieeexplore.ieee.org/document/8278802/>. Acesso em: 20 jun. 2018.

AGUIAR, Fernando Ferreira et al. Desenvolvimento e implantação de um Fab Lab: um estudo teórico. Revista Espacios Vol. 38 (No 31) 2017. Pág. 1 ISSN $0798 \quad 1015 . \quad$ Disponível em: <www.revistaespacios.com/a17v38n31/a17v38n31p01.pdf>. Acesso em: 28 jun. 2018.

BASMER, S. et al. Open production: chances for social sustainability in manufacturing. Procedia CIRP. Vol. 26, 2015, Pages 46-51. Disponível em: <https://doi.org/10.1016/j.procir.2014.07.102>. Acesso em: 04 jun. 2018.

BASTOS, Victória Fernandez. Moda e Fabricação Digital em um contexto Fab Lab: equipamentos, métodos e processos para o desenvolvimento de produtos. Dissertação (Mestrado em Design) - Universidade Federal De Pernambuco - Centro de Artes e Comunicação - Programa de Pós-graduação em Design. Recife, p. 153. 2014. Disponível em: <http://www.proceedings.blucher.com.br/article-details/moda-e-

fabricao-digital-em-um-contexto-fab-lab-equipamentos-mtodos-e-processospara-o-desenvolvimento-de-produtos-24628>. Acesso em: 25 jun. 2018

BEN REJEB, Helmi. ROUSSEL, Benoît. Design and Innovation Learning: case study in north african engineering universities using creativity workshops and fabrication laboratories. 28th CIRP Design Conference, 2018. Disponível em: <https://doi.org/10.1016/j.procir.2018.03.263>. Acesso em: 25 maio 2018.

BORGES, Karen.; PERES, André; CASTILHO, Maria; DA CRUZ, Léa Fagundes. Possibilidades e desafios de um Espaço Maker com objetivos educacionais. Tecnologia Educacional. 1. 22 - 32, 2015. Disponível em: <http://abt-br.org.br/wp-content/uploads/2017/03/210.pdf> Acesso em: 25 maio 2018.

BULL, Glen et al. Make to learn: invention through emulation. Smart Learning Environments, 2017. 4:8 DOI 10.1186/s40561-017-0047-5. Acesso em: 25 maio 2018. 
COSTA, Christiane Ogg; PELEGRINI, Alexandre Vieira. O design dos Makerspaces e dos Fab Labs no Brasil: um mapeamento preliminar. Design e Tecnologia, [S.l.], v. 7, n. 13, p. 57-66, jun. 2017. ISSN 2178-1974. Disponível em: <https://www.ufrgs.br/det/index.php/det/article/view/375>. Acesso em: 13 jun. 2018.

CRESWELL, John W. Projeto de pesquisa: métodos qualitativo, quantitativo e misto. 6 ${ }^{\mathrm{a}}$ Ed. São Paulo, SP: Pearson Prentice Hall, 2007.

DE CAMPOS, Paulo Eduardo Fonseca; DIAS, Henrique José dos Santos. A insustentável neutralidade da tecnologia: o dilema do Movimento Maker e dos Fab Labs. Disponível em: < https://doi.org/10.18617/liinc.v14i1.4152>. Acesso em: 13 jun. 2018.

DRESCH, A.; LACERDA, D. P.; ANTUNES JÚNIOR, J. A. V. Design science research: método de pesquisa para avanço da ciência e tecnologia. Porto Alegre: Bookman, 2015.

FROSCH, Renato; ALVES, Antônio Fernando Gomes. Perspectivas para a formação docente universitária com aspectos Makers. Revista de Estudos Aplicados em Educação, v. 2, n. 4, jul./dez. 2017. Disponível em: $<10.13037 /$ rea-e.vol2n4.4997>. Acesso em: 14 jun. 2018.

GAEIRAS, Bernardo. Fab Lab Lisboa: when a municipality fosters grassroots, technological and collaborative innovation. Field Actions Science Reports [Online], Special Issue 16 | 2017, Online since 01 June 2017, connection on 09 January 2018. Disponível em: <http://journals.openedition.org/factsreports/4304>. Acesso em: 28 maio 2018

GALVÃO, Taís Freire; PEREIRA, Maurício Gomes. Revisões sistemáticas da literatura: passos para sua elaboração. Disponível em: <http://www.scielo.br/pdf/ress/v23n1/2237-9622-ress-23-01-00183.pdf>. Acesso em: 25 maio 2018.

GIUISTI, Jessica D.; ALBERTI, Fernando G.; BELFANTI, Federica. Makers and clusters: knowledge leaks in open innovation networks. Journal of Innovation \& Knowledge (2017). Disponível em: <https://doi.org/10.1016/j.jik.2018.04.001>. Acesso em: 28 maio 2018.

KOHTALA, Cindy. Making "Making" Critical: how sustainability is constituted in Fab Lab ideology. The Design Journal, 20:3, 375-394, Disponível em: <DOI:10.1080/14606925.2016.1261504>. Acesso em: 18 jun. 2018.

MARAVILHAS, Sérgio; MARTINS, Joberto. Strategic knowledge management a digital environment: tacit and explicit knowledge in Fab Labs. Journal of Business Research (2018), Disponível em: <https://doi.org/10.1016/j.jbusres.2018.01.061>. Acesso em: 25 jun. 2018.

NASCIMENTO, Susana; PÓLVORA, Alexandre. Maker cultures and the prospects for technological action. Sci Eng Ethics (2018) 24:927-946 
Disponível em <https://doi.org/10.1007/s11948-016-9796-8>. Acesso em: 27 maio 2018.

NETO, Emílio Bertholdo. Sistemas de tecnologia urbanas colaborativas: os casos da Rede Fab Lab Livre SP e do MobiLab. 2018, 247f. Disponível em: <http://tede.mackenzie.br/jspui/handle/tede/3583>. Acesso em: 11 jun. 2018.

NEVES, Heloísa. Maker Innovation: do open design e fab labs ... às estratégias inspiradas no movimento maker. São Paulo, 2014, 261 p. Disponível em: <http://www.teses.usp.br/teses/disponiveis/16/16134/tde14072015-112909/pt-br.php>. Acesso em: 30 jun. 2018.

OLIVEIRA, Diego Jucá de Lima. 0 uso da Prototipagem e Fabricação Digital no ambiente Fab Lab. 2016. 109 f. Disponível em: <http://hdl.handle.net/10183/142793>. Acesso em: 11 jun. 2018.

RUIZ, Maria Elena García; ACEBO, Francisco Javier Lena. Fab Lab global survey characterization of Fab Lab phenomenon. Disponível em: <https://ieeexplore.ieee.org/iel7/8390719/8398632/08399154.pdf>.

Acesso em: 21 jun. 2018.

SANTANA, André L. M. et al. Lite Maker: um fab lab móvel para aplicação de atividades mão na massa com estudantes do ensino básico. Disponível em: <http://dx.doi.org/10.5753/cbie.wie.2016.21>. Acesso em: 12 jun. 2018.

SAVASTANO, Marco et al. Fab Labs as Platforms for Digital Fabrication Services: a literature analysis. IESS 2017, LNBIP 279, pp. 24-37, 2017. Disponível em: <DOI: 10.1007/978-3-319-56925-3_3>. Acesso em: 27 jun. 2018.

STACEY, Michael. The Fab Lab network: a global platform for digital invention, education and entrepreneurship. Innovations: Technology, Governance, Globalization Volume 9 | Issue 1-2 | Winter-Spring 2014. Disponível em: <https://doi.org/10.1162/inov_a_00211>. Acesso em: 28 maio 2018.

URRUTIA PINTO, Sofia Lorena et al. O Movimento Maker: enfoque nos Fab Labs brasileiros. Disponível em: <http://www.anprotec.org.br/moc/anais/ID_147.pdf>. Acesso em: 25 maio 2018.

URRUTIA PINTO, Sofia Lorena et al. Fab City com enfoque em economia circular. Congresso Nacional de Inovação e Tecnologia, [S.I.], oct. 2017. ISSN 2526-3145.

Disponível

em: <http://www.inova.ceplan.udesc.br/index.php/inova/ article/view/59>. Acesso em: 02 ago. 2018.

VIEIRA, Regiane Balestra; BRESCIANI, Luis Paulo; DOS SANTOS, Isabel Cristina. Fab Labs network in developing countries knowledge: spillover effects or managing technology development within the scarcity economy? 2017 Proceedings of PICMET '17: Technology Management for Interconnected World. Disponível em: 
<https://ieeexplore.ieee.org/document/8125265/>. Acesso em: 15 jun. 2018.

VOIGT, Christian; UNTERFRAUNER, Elisabeth; STELZER, Roland. Diversity in Fab Labs: culture, role models and the gendering of making. Springer International Publishing AG 2017 I. Kompatsiaris et al. (Eds.): INSCI 2017, LNCS 10673, pp. 52-68, 2017. Disponível em: <https://doi.org/10.1007/978-3-319-70284-1_5>. Acesso em: 27 jun. 2018.

YIN, R. K. Estudo de caso: planejamento e métodos. Porto Alegre: Bookman, 2001. 2. ed. 